\title{
Amarração de poços a dados sísmicos utilizando perfil de resistividade, na Bacia do Parnaíba
}

Raquel Macedo Dias - Universidade Federal Fluminense

André Lopes Ferreira - Agência Nacional do Petróleo, Gás Natural e Biocombustíveis.

Copyright 2018, SBGf - Sociedade Brasileira de Geofísica

Este texto foi preparado para a apresentação no VII Simpósio Brasileiro de Geofísica, Salinópolis, 18 a 20 de setembro de 2018. Seu conteúdo foi revisado pelo Comitê Técnico do VII SimBGf mas não necessariamente representa a opinião da SBGf ou de seus associados. É proibida a reprodução total ou parcial deste material para propósitos comerciais sem prévia autorização da SBGf.

\section{Resumo}

Uma das maiores dificuldades na exploração de petróleo na Bacia do Parnaíba é a escassez de dados sísmicos e de poços. Neste trabalho, desenvolveu-se uma metodologia para a amarração do poço 2BGST0001 MA, que, em razão de ter sido perfurado nos anos iniciais de exploração da bacia, não possui perfis básicos como sônico e densidade, mas possui perfil de resistividade. Através da aplicação das relações de Gardner e Faust no perfil de resistividade, foi possível criar o traço sintético para a amarração com a sísmica. Com o suporte da relação tempo-profundidade do poço $1 \mathrm{FL} 0001 \mathrm{PI}$, foi realizada uma correção de drift para uma estimativa inicial do posicionamento do sintético com relação à sísmica. Todo o fluxo de trabalho foi feito em Python, sendo totalmente reproduzível.

\section{Introdução}

A Bacia do Parnaíba está localizada na região nordeste ocidental do território brasileiro, com sua maior porção no Estado do Maranhão, abrangendo $6 \times x .000 \mathrm{~km}^{2}$. Os Sistemas Petrolíferos desta bacia estão associados com a intrusão de diques e soleiras de diabásio, responsáveis pelo aporte térmico necessário, à geração de gás natural. A exploração de gás na bacia se iniciou na década de 1950, e hoje existem sete campos exploratórios, que compõem o Parque dos Gaviões. A bacia ainda é pouco explorada, contando com 99 poços exploratórios, $15098,69 \mathrm{~km}$ lineares de sísmica bidimensional e 462,45 $\mathrm{km}^{2}$ de sísmica tridimensional.

A amarração de dados de poços, escassos na Bacia do Parnaíba, é um grande desafio para os exploracionistas. Isso se deve ao fato de que muitos dos poços disponíveis foram perfurados nos anos iniciais da exploração da bacia e não possuem perfis básicos como sônico e densidade, apresentando apenas perfis de potencial espontâneo e resistividade. Devido a esta escassez de dados, desenvolveu-se neste trabalho uma metodologia para amarrar poços utilizando apenas o perfil de resistividade, as relações de Gardner e Faust e, para a correção de drift, a Relação Tempo-Profundidade (Time Depth Relationship, TDR) de um poço próximo. Todo o trabalho foi realizado em Python (ferramenta opensource) sendo totalmente reproduzível. O Notebook do
Jupyter com o código para reproduzir este trabalho pode ser baixado em https://github.com/raqmac/VIII_SimBGf.

O foco deste trabalho é amarração do poço 2BGST0001 MA, perfurado em 1959, que possui somente dados de resistividade. Este poço, que neste trabalho chamamos de "poço alvo" da amarração, encontra-se próximo à linha sísmica 0317-0112 do levantamento 0317_ANP_BACIA_DO_PARNAIBA. Nos arredores, (39,3 km a leste do poço alvo) localiza-se o poço $1 \mathrm{FL} 0001 \mathrm{PI}$, que possui perfil sônico e foi amarrado à linha sísmica 0317-0113, de acordo com os procedimentos tradicionais. A TDR deste poço foi utilizada em seguida em uma segunda amarração, para a correção de drift.

\section{Metodologia/ Problema Investigado}

2.1 Criação de traço sintético a partir de um perfil de resistividade.

A sísmica de reflexão é a principal ferramenta de exploração geológica para prospecção de petróleo. Para a sua correta utilização é imprescindível relacionar as reflexões sísmicas com interfaces geológicas. O processo utilizado para isso é o de gerar um modelo unidimensional para a transmissão da energia sísmica através da geologia do poço em análise, o traço sintético.

Para a criação do traço sintético, primeiramente calculouse um perfil de velocidade (P) a partir do perfil de resistividade do poço 2BGST0001 MA, utilizando a relação de Faust:

$$
V_{p}=\gamma(Z F)^{\frac{1}{6}}
$$

onde $V_{p}$ é a velocidade em $\mathrm{km} / \mathrm{s}, \gamma$ é uma constante $\gamma=2.2888, Z$ é a profundidade (em MD) em km e $F$ é o fator de formação da resistividade $F=R_{t} / R_{w}$, sendo $R_{t}$ a resistividade profunda e $R_{w}$ a resistividade da água (Simm e Bacon, 2014). Utilizou-se o perfil de resistividade do poço como os valores de resistividade profunda.

Com o perfil de $V_{p}$, calculou-se um perfil de densidades, utilizando a relação de Gardner:

$$
\rho_{b}=d V_{p}^{f}
$$

onde $\rho_{b}$ é a densidade bulk, e $d$ e $f$ são constantes. A velocidade utilizada na relação de Gardner foi calculada a partir da relação de Faust. As constantes utilizadas foram $f=0.3$ e $d=1.75$ (Simm e Bacon, 2014).

Com os perfis de Velocidade e Densidade prontos, foi possível criar o traço sintético com o seguinte fluxo de trabalho: 
i. Cálculo do perfil de impedância, utilizando a relação $I=\rho V$ e os perfis de Velocidade e densidade calculados anteriormente.

ii. Cálculo do perfil de refletividade, utilizando a relação $R=\frac{I_{2}-I_{1}}{I_{2}+I_{1}}$

iii. Criação da relação tempo $\mathrm{x}$ profundidade, a partir do perfil de velocidade calculado anteriormente.

iv. Reamostragem do perfil de impedância para $4 \mathrm{~ms}$. Como os dados de poço possuem resolução muito alta, foi necessário fazer a reamostragem para a mesma taxa da sísmica (4ms) e também a aplicação de um filtro passabaixa (Smith, 1997). A reamostragem e a filtragem foram feitas no perfil de Impedância, e o perfil de refletividade foi recalculado a cada passo.

v. Convolução do perfil final de Refletividade com uma wavelet Ricker de frequência central de $25 \mathrm{~Hz}$, estimada a partir da sísmica, com o perfil final de Refletividade.

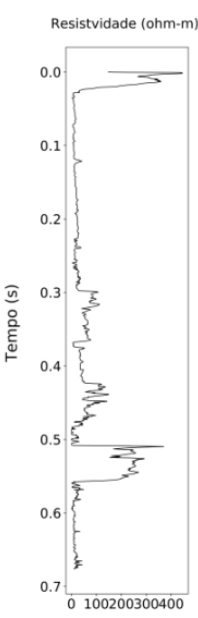

(a)

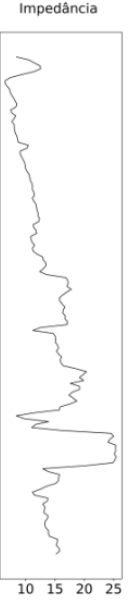

(b)

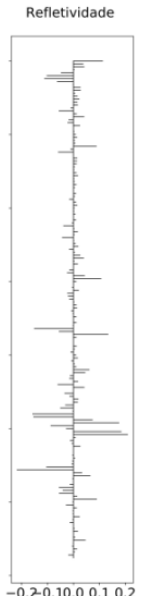

(c)

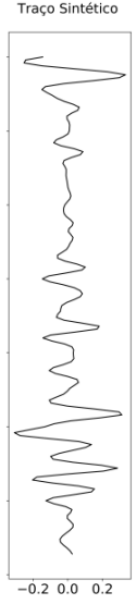

(d)
Figura 1. Criação de traço sintético a partir de resistividade. (a) Resistividade original do poço. (b) Impedância calculada a partir das relações de Gardner e Faust. (c) Refletividade calculada a partir da Impedância. (d) Traço Sintético calculado a partir da convolução da refletividade com a wavelet Ricker.

\subsection{Correção de drift}

A correção de drift é utilizada para combinar a informação de velocidade do perfil sônico com a de um levantamento de Vertical Seismic Profile (ou VSP, normalmente zerooffset, conhecido como checkshot). O objetivo é obter um perfil de velocidade (de ondas compressionais) mais representativo da região do poço, e dessa forma fazer um melhor ajuste do poço-sintético à linha sísmica.

Em um fluxo padrão, esta correção é feita utilizando VSP como referência para ajuste do sônico no próprio poço. Neste trabalho, porém, devido à falta de checkshot no poço alvo, utilizou-se a TDR do poço próximo 1FL 0001
PI como referência. Seguiu-se o seguinte fluxo de trabalho para a correção de drift:

i. Integração do perfil de velocidades do poço alvo e comparação com a TDR do poço próximo.

ii. Cálculo do drift, subtraindo a integral do perfil de velocidades do poço alvo da TDR do poço próximo.

iii. Ajuste de segmentos de linha como tendência nos pontos do drift.

iv. Aplicação da tendência ajustada ao drift sobre a integral do perfil de velocidades do poço alvo. Esta correção pode ser aplicada (somada) tanto para ajustar a TDR quanto para ajustar diretamente o perfil de velocidades do poço alvo (correção de velocidade).

\section{Resultados}

\subsection{Primeira Amarração: Apenas Resistividade}

Para avaliar a primeira amarração, foi criado um display do sintético sobre a sísmica, com 300 traços laterais. Devido ao datum de processamento sísmico ter sido estabelecido em $600 \mathrm{~m}$ acima do nível do mar e 340 metros acima da cabeça do poço, foi necessário aplicar um shift (deslocamento) em tempo no sintético. Para este cálculo, foi utilizada a velocidade de substituição de 3000 $\mathrm{m} / \mathrm{s}$ do processamento sísmico.

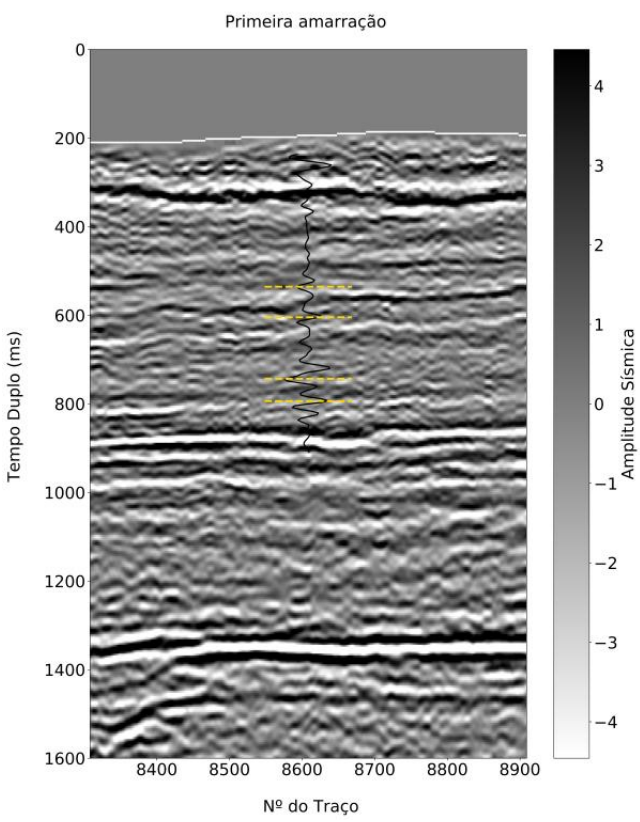

Figura 2. Amarração do sintético derivado da resitividade, utilizando uma janela da sísmica com 300 traços para cada lado. As linhas amarelas tracejadas correspondem ao topo e base das soleiras identificadas na litologia do perfil composto.

\subsection{Segunda Amarração: Correção das velocidades com tendência de TDR de Poço Próximo.}

Como a primeira amarração não atingiu o resultado desejado, foi adicionada uma tendência linear de velocidades a partir de um poço próximo. Vale ressaltar 
que, devido às características de bacia intracratônica sag do Parnaíba, é razoável assumir que uma tendência de $1^{\mathrm{a}}$ ordem (linear) de velocidades seja respeitada regionalmente à distância desses poços.

A adição desta tendência linear foi feita a partir da correção do drift (Seção 2.2), utilizando como referência a TDR do poço 1FL $0001 \mathrm{PI}$. Podemos ver o resultado do drift calculado na figura 3.

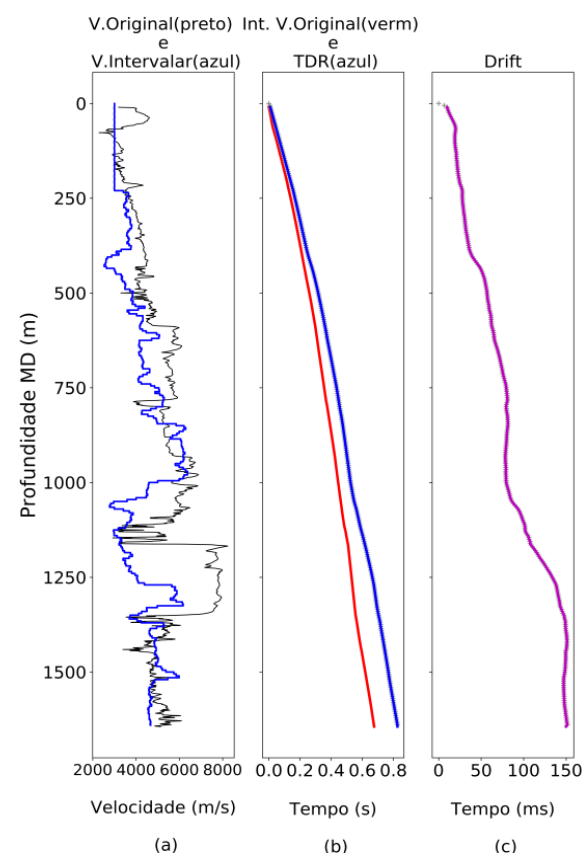

Figura 3. a) Perfil de velocidades do poço alvo (preto) e do poço próximo (azul); b) Integral do perfil de velocidades do poço alvo (vermelho) e TDR do poço próximo (azul); c) Drift entre a e $b$.

Para obter a tendência regional linear de velocidades, fitou-se uma função linear sobre o drift. Esta função linear representa a tendência regional ou "background" de velocidades do poço alvo. Neste trabalho somou-se a tendência do drift sobre a TDR prévia do poço alvo (figura 4). Na figura 5 estão os resultados da correção do drift aplicada ao perfil de velocidades.

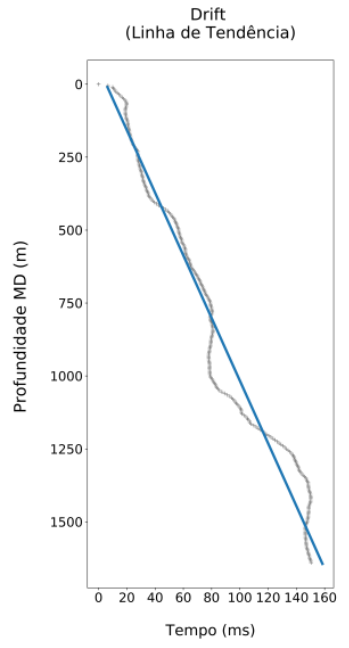

(a)
Int. V.Original(verm) Int. V.Corrigida(verde)

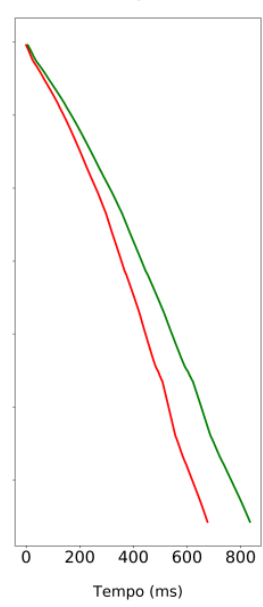

(b)
Figura 4. a) Drift calculado (cinza) e ajuste linear da tendência do drift(azul); b) Integral do perfil de velocidades alvo antes (vermelho) e depois (verde) da aplicação da tendência do drift;

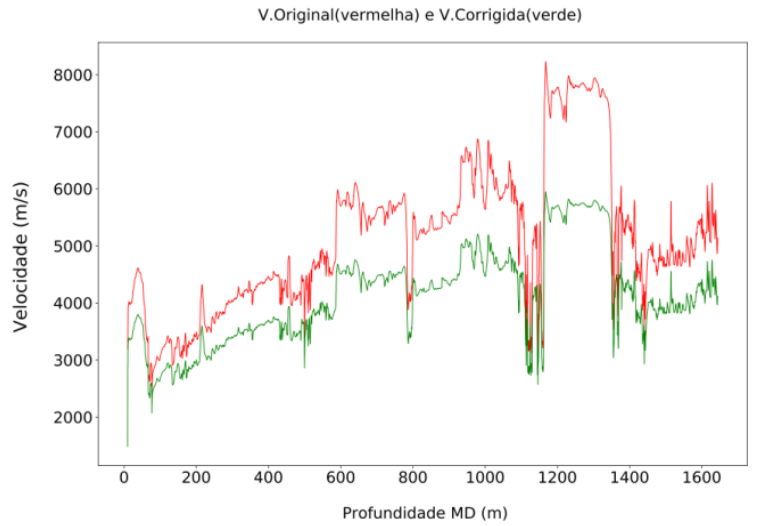

Figura 5. Perfil de velocidades do poço alvo antes (vermelho) e depois (verde) da correção do drift.

A partir desta correção, foi refeito o fluxo da seção 2.1 para a criação de um novo sintético. Neste caso utilizouse o mesmo perfil de densidade, porém em vez de calcular uma nova TDR (como diz o item 3 deste mesmo fluxo) utilizou-se a TDR corrigida de drift, repetindo os itens $1,2,4$ e 5 do fluxo.

Para esta segunda amarração, foi criado o mesmo display utilizado na seção 3.1 para o sintético previamente calculado. Os limites das soleiras estão plotados em rosa tracejado na figura 6 .

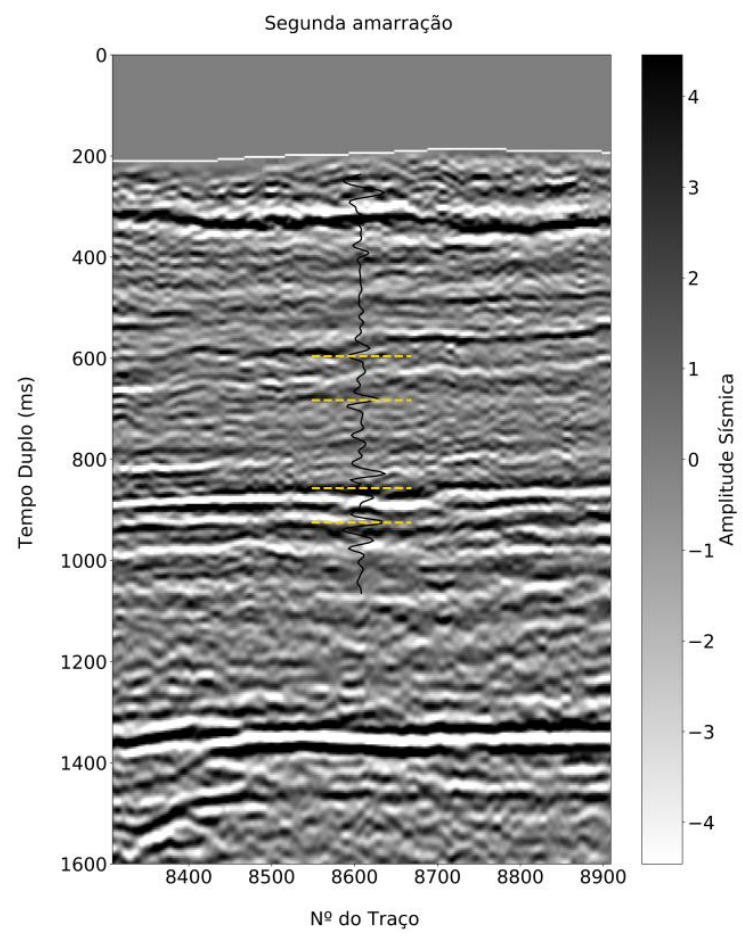

Figura 6. Amarração do sintético após a correção de drift, utilizando uma janela da sísmica com 300 traços para cada lado. As linhas amarelas tracejadas correspondem ao topo e base das soleiras identificadas na litologia do perfil composto. 


\section{Discussão e Conclusões}

Ao analisar a primeira amarração sobre a sísmica, assumiu-se que as soleiras estariam localizadas nas reflexões de maior contraste, porém os limites das soleiras (plotados em amarelo tracejado nas figuras $7.1 \mathrm{e}$ 7.2) não combinavam com estas reflexões mais fortes. Comparando com outros poços próximos, concluiu-se que as velocidades no poço alvo estavam muito maiores do que o esperado. No poço próximo, as velocidades das soleiras, por exemplo, estão na casa de 5200 à $6000 \mathrm{~m} / \mathrm{s}$, porém, analisando o perfil de velocidades do poço alvo, percebeu-se que a velocidade da segunda soleira estava próxima de $8000 \mathrm{~m} / \mathrm{s}$. Esta grande diferença nas velocidades das soleiras junto com a análise do corredor de traços levou à conclusão de que, apesar de as aproximações de Gardner e de Faust serem eficazes para estimar 0 contraste entre as interfaces, (representado pelos coeficientes de reflexão), elas não foram suficientes para posicionar corretamente estes contrastes em relação à sísmica.

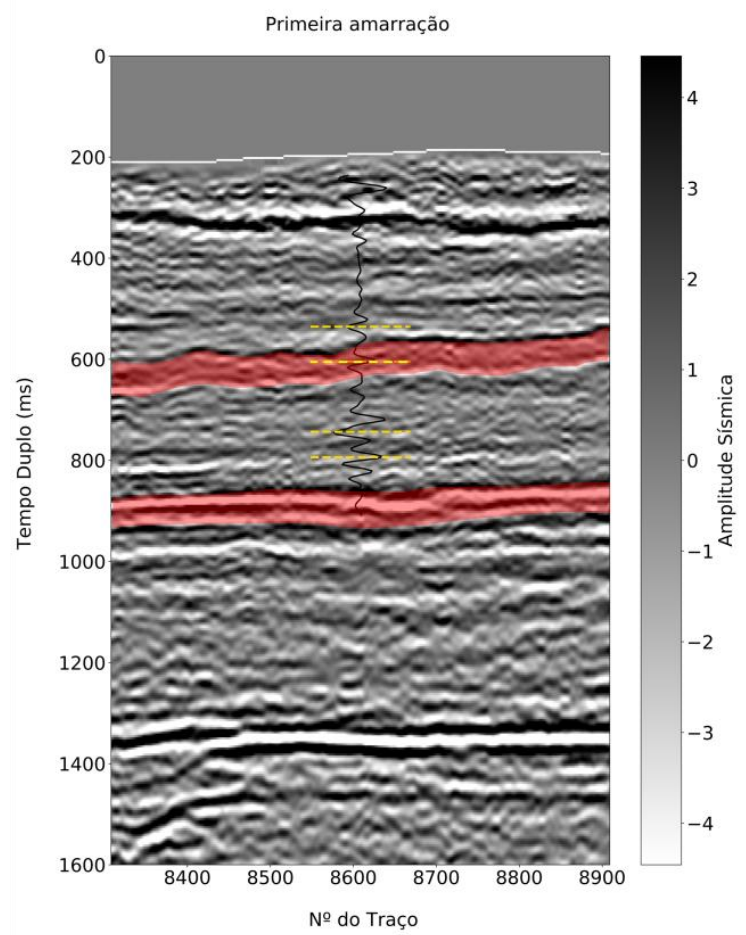

Figura 7.1. Primeira amarração, com a interpretação das soleiras. O sintético "encurtado" em relação às posições das soleiras evidencia as velocidades muito altas do poço alvo.
Dessa forma, decidiu-se corrigir a TDR do poço alvo utilizando a TDR do poço 1FL0001 PI para adicionar uma tendência linear regional de velocidades. Ao interpretar a segunda amarração sobre o corredor de traços, constatou-se que as soleiras indicadas pelo sintético estavam mais bem localizadas com relação às reflexões de maior contraste de amplitude. Desta vez, também, as velocidades no poço alvo estavam mais condizentes com a realidade: analisando o perfil de velocidades do poço alvo após a correção do drift, as velocidades da primeira e da segunda soleira ficaram próximas a $4500 \mathrm{~m} / \mathrm{s}$ e $5600 \mathrm{~m} / \mathrm{s}$, respectivamente.

As figuras 7.1 e 7.2 mostram os resultados das duas amarrações, juntamente com uma interpretação das respectivas soleiras.

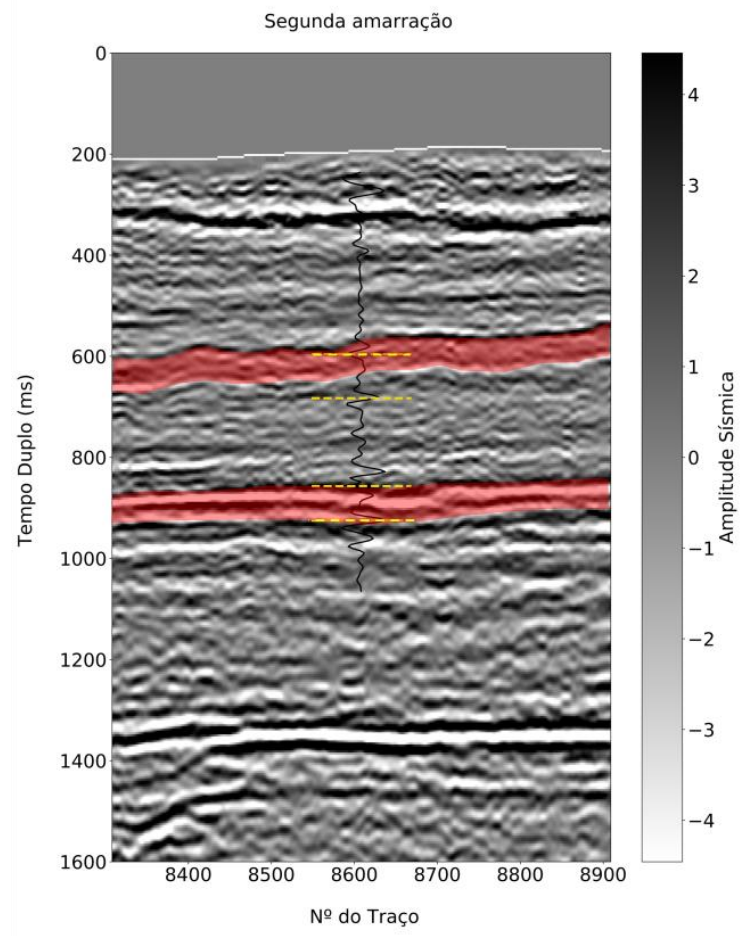

Figura 7.2. Segunda amarração, com a interpretação das soleiras. Aqui, o posicionamento do traço sintético está mais condizente com as posições das soleiras. 
Com o sintético e a TDR obtidos, pode-se aprofundar a amarração através de ferramentas como: análise de correlação traço sísmico x sintético, ajustes de time-shift, strech and squeeze. Recomenda-se, nesta etapa, utilizar um overlay do envelope com a própria linha sísmica, para uma melhor visualização dos contrastes de energia. Esta ferramenta pode ser útil para análise de possíveis distorções de fase esperadas devido aos fenômenos de dispersão de onda, comum em dados terrestres. $\mathrm{Na}$ figura 8 há um exemplo do display do envelope sobre a linha sísmica.

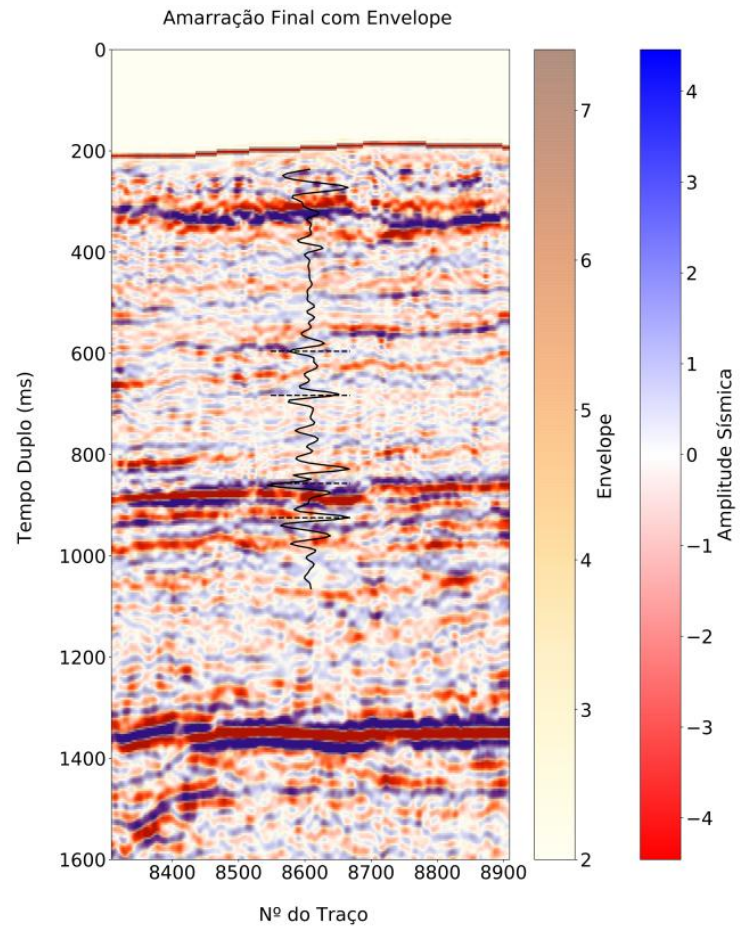

Figura 8. Análise do atributo envelope sobre a sísmica, com o traço sintético após a correção de drift. As linhas pretas tracejadas representam o topo e a base das soleiras identificadas na litologia do perfil composto.

Por fim, conclui-se que, para poços com ausência de informações de velocidade, que tenham apenas o perfil de resistividade, as aproximações de Gardner e Faust, se mostram suficientemente eficazes para estimar 0 contraste entre as interfaces e, em conjunto com a correção de drift, também podem auxiliar numa estimativa inicial do posicionamento destes contrastes com relação à sísmica.

A metodologia aqui apresentada também pode ser aplicada em outras bacias sedimentares que apresentem as mesmas dificuldades em relação à ausência de dados de poços. As bacias do Amazonas e Paraná, por exemplo, também carecem de dados de poço em quantidade, além de apresentarem características geológicas similares à Bacia do Parnaíba, como a presença de grandes corpos magmáticos intrusivos.

\section{Agradecimentos}

Agradecemos à ANP pelo suporte e permissão para publicar estes resultados e à Cimageo pela disponibilização dos dados reprocessados do levantamento 0317_ANP_BACIA_DO_PARNAIBA, em especial ao geofísico Wañder Amorim pelas preciosas discussões, assim como ao geofísico Rodrigo Morelatto, da ANP.

\section{Referências}

Simm, R., Bacon M., 2014. Seismic Amplitude: An Interpreter's Handbook. Cambridge University Press p.38-57.

Smith, S.W., 1997. The Scientist and Engineer's Guide to Digital Signal Processing - p.55.

Henry, G., 1994. Geophysics for Sedimentary Basins. Éditions Technip - p. 328-339.

Cunha, F.M.B. 1986. Evolução paleozoica da Bacia do Parnaíba e seu arcabouço tectônico. 107f. Dissertação (Mestrado em Geologia) - Programa de Pós-graduação em Geologia, Instituto de Geociências, Universidade Federal do Rio de Janeiro, Rio de Janeiro. 